\title{
THE EFFECT RICKER WAVELET OF DUTY CYCLE ADJUSTMENT ON GPR DETECTION RESULT
}

\author{
Aditya Rifky Ramadhan ${ }^{1}$, Erfansyah Ali $^{2}$, A.A.Pramudita ${ }^{3}$ \\ ${ }^{1,2,3}$ School of Electrical Engineering, Telkom University \\ ${ }^{1}$ rifkyfaris2015@gmail.com, ${ }^{2}$ erfansyahali@telkomuniversity.ac.id, \\ ${ }^{3}$ pramuditaadya@telkomuniversity.ac.id
}

Manuscript received April 16, 2021; revised May 22, 2021; accepted June 10, 2021

\begin{abstract}
Ground Penetrating Radar (GPR) employs an ultra-wideband (UWB) signal for detecting objects under the ground surface. In a certain GPR application, a proper UWB signal is needed to obtain a good detection result. Ricker wavelet is one type of UWB signal that can be used in GPR operation. The effect of adjusting the Ricker wavelet duty cycle on the B-scan result was investigated and the result is discussed in this paper. Laboratory experiments were performed by modelling the GPR system using Vector Network Analyzer (VNA). The result shows that selecting a Ricker wavelet's duty cycle is successful to show the target clearly.
\end{abstract}

Keywords: GPR, Ricker wavelet, VNA, duty cycle.

DOI: $10.25124 /$ jmecs.v8i1.2872

\section{Introduction}

Buried object detection is one of several engineering research. It has a huge range of applications, such as ground exploration, buried mines detection, cable repair, cable maintenance, pipe repair, and many more. [1, 2]. More detailed functions of GPR are for concrete thickness [3, 4], void detection, and corrosion. GPR system include an antenna, amplifier, signal processing, display, and many more. The function of the antenna is to transmit and receive electromagnetic waves to and from the ground. The received electromagnetic waves from the antenna are forwarded to the signal processing to convert the sinusoidal signal into an image. The accumulated signals are displayed and stored as data and images on the computer $[5]$.

In geo-radar, the method of generating seismic data with a pulse shape and a good signaling process requires a good method, one of the best is the Ricker wavelets $[6,7]$. Ricker wavelets is theoretically a solution to the Stokes differential equation that can be applied to propagated seismic waves
[8]. Ricker wavelets needs to be approximated to find the best detection results by improving resolution to increase detection results $[7,9]$. One way to improve the resolution is to find the best duty cycle.

This paper describes a Fast Fourier Transform (FFT) of Ricker's pulses with received signal from a vector network analyzer (VNA). The results are transformed into a B-scan so that the target becomes easier to recognize. There are three forms of the pulse with the simulated duty cycle of $0.585 \%, 3.42 \%$, and $9.52 \%$. These three results are compared and observed to find the best result that shows the target shape as clearly as possible. Another benefit of this study is knowing the appropriate pulse width for the best configuration in GPR.

\section{Pulse Shaping in GPR}

\subsection{GPR System}

In Fig. 1, briefly describe the GPR system that consists of the display, transmitter (Tx), re- 
ceiver $(\mathrm{Rx})$, Tx antenna, and $\mathrm{Rx}$ antenna. $\mathrm{Tx}$ is connected to the Tx antenna to transmit electromagnetic waves to the ground to detect underground targets [5]. The electromagnetic waves that hit the target are reflected to the $\mathrm{Rx}$ antenna. The $\mathrm{Rx}$ antenna is connected to the $\mathrm{Rx}$ to process the received signal. In GPR, there are three reflections produced from the transmission of electromagnetic waves symbolized by $\mathrm{S} 1, \mathrm{~S} 2$, and $\mathrm{S} 3$ which are a reflection provided by the receiving antenna, a reflection of the ground surface, and a reflection of the target [3], respectively. According to a generally accepted terminology, the GPR data is divided into three, namely A-scan, B-scan, and C-scan.

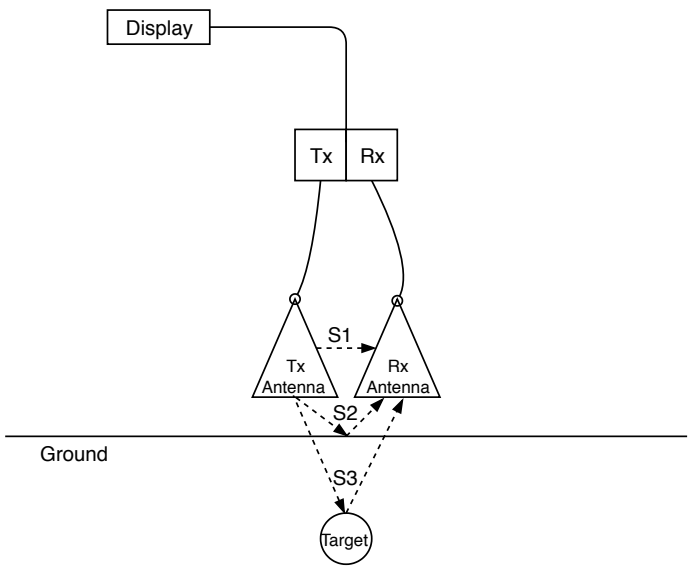

Fig. 1. GPR System Signals Modelling.

A-scan is the trace of a single signal or spatial point recorded in the form of two-dimensional data by a GPR receiver. The primary value of the A-scan is the monitoring of quality control data which may include signal reflections of the target [5] [10]. The A-scan scheme takes only one point and results where three reflections occur as three sums exist in Eq. (1). Where $S_{r}, S_{t}, r_{1} S_{t}$, $r_{2} S_{t}$ is a received signal, a transmitted signal, a ground-surface reflection, and a reflection of target [3], respectively.

$$
S_{r}(t, X)=S_{t}+r_{1} S_{t}\left(t-\alpha_{1}\right)+r_{2} S_{t}\left(t-\alpha_{2}\right)
$$

B-scan is two-dimensional data, referring to the fact that every B-scan consists of a collection of A-scans, which includes one scanned path [10] [5]. If a target occurs, B-scan forms a hyperbole. $\mathrm{B}$-scan is only a color image if there is no target [11]. The B-scan scheme takes multiple A-scans in a single path found in Eq. (2)

$$
S_{B}=\left[S_{r}\left(t, X_{1}\right) S_{r}\left(t, X_{1}\right) \ldots S_{r}\left(t, X_{n}\right)\right]
$$

where $S_{B}$ contains a B-scan containing several $S_{r}(t, X)$, A-scan.

\subsection{Pulse Shaping}

The pulse shaping technique is to optimize power, attenuation, and bandwidth using an ultrawideband (UWB) signal. Overall, the structure of pulses can affect the shape of the target displayed on the B-scan. The resolution range $(\Delta r)$ depends on the GPR bandwidth that generated and calculated as shown in Eq. (3), and bandwidth depends on the pulse width that generated as shown in Eq. (4)

$$
\begin{gathered}
\Delta r=\frac{v}{2 B} \\
B=\frac{1}{\tau_{p}}
\end{gathered}
$$

where $v$ is the speed of electromagnetic waves in the ground, $B$ is the bandwidth and $\tau_{p}$ is the pulse width[6]. Equation (3) and (4) imply that in order to gain large resolution it requires small bandwidth with a large pulse width.

The Ricker wavelet is one of the UWB signals used in pulse shaping. It is a Stokes differences equation solution that takes into consideration the effect of Newtonian viscosity which applies to seismic waves spread by homogeneous media [8]. Ricker wavelet is a time-domain waveform illustrated in Fig 2. Generalized wavelets are mathematically defined as Gaussian function fractional derivatives [12]. The Ricker Wavelet in the time domain defined as shown in Eq. (5)[8]

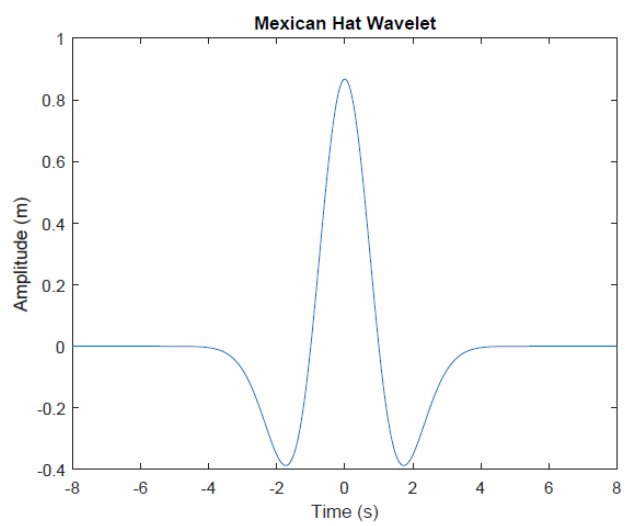

Fig. 2. Ricker Wavelet Pulse Shape.

$$
r(\tau)=\left(1-\frac{1}{2} \omega^{2}{ }_{p} \tau^{2}\right) \exp \left(-\frac{1}{4} \omega^{2}{ }_{p} \tau^{2}\right)
$$


Ramadhan et al / Journal of Measurement, Electronics and Communication Systems

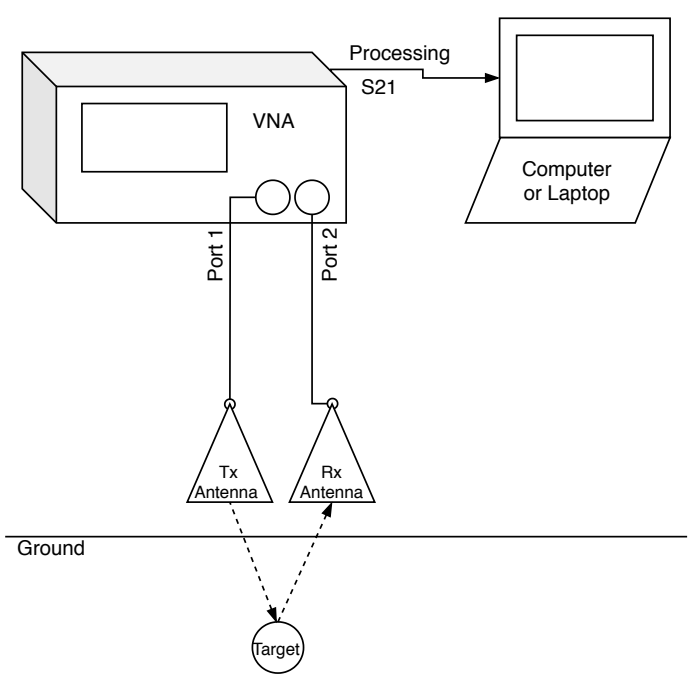

Fig. 3. GPR Modelling Using VNA.

where $\tau$ is the time (in seconds) and $\omega$ is the most intense frequency (in radians per second), which can affect the width of the pulse in one period.

\subsection{GPR Modelling Using VNA}

VNA is being used to analyze networks with high precision and accuracy where ultra-wideband transmitters and receivers with a frequency range are appropriate for communication technology needs. The transmitter is the VNA port-1, while the receiver is port- $2[3,13]$. Fig. 3 displays the data retrieval process using VNA which allows electromagnetic wave transmission through the Tx anntena. After hitting the target, the Rx antenna receives the signals which subsequently are displayed on the VNA monitor.

Data generated by $S_{21}$ will be processed by software. After the processing in software, $S_{21}$ is transformed into $\mathrm{H}(\mathrm{f})$ and used to calculate the received waveform as written in Eq.(6)

$$
S r(f)=S t(f) H(f)
$$

where $H(f)$ is a delay propagation that a received signal, $S_{t}(f)$ is a transmitted waveform, and $S_{r}(f)$ is the received waveform that shown in the display which is the final result of the multiplication. $S_{t}(f)$ can be changed for the pulse shape.

\section{Experiment Setup}

The experiment has seven steps to complete data retrieval. The first step is to place the table near to the sandbox with the VNA on the table. The second step is to mount the antenna into the stand with the distance between the antennas at $8 \mathrm{~cm}$ and the distance from the ground at $12 \mathrm{~cm}$. The third step is to connect the antenna to the
VNA using a coaxial cable. The fourth step is to place the antenna in the sandbox. The sixth step is to change the measurement to $S_{21}$ and adjust the data format to a complex number. The final step is to save VNA data with the save trace data button.

FFT receives a signal from VNA and displays the signal as A-scan data using the software. If the A-scan data is incorrect, the data retrieval is performed again to obtain the desired data. When all A-scan data has been obtained, the next step is to process A-scan data with Ricker wavelet and several duty cycles and then continue to process Ascan data in a B-scan. On the B-scan, it is enough to see the target while the A-scan is difficult to see the target.

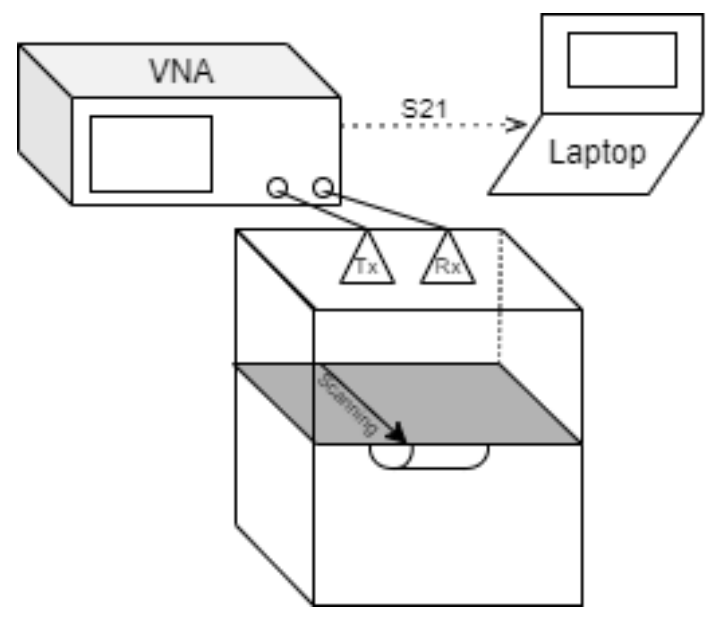

Fig. 4. Experiment Design.

Measurements is made using a VNA connected to the antenna. In Fig 4, the sandbox is placed outside the room and made from wood with $(1 \mathrm{x} 1 \mathrm{x} 1)$ $\mathrm{m}$ dimension. The wood is a dielectric material, so it assumed not affect the final results of the data. The target used is a can with a height of 13 $\mathrm{cm}$ and a diameter of $5 \mathrm{~cm}$. The sandbox is half full, as shown in Fig. 4. The can is placed at a depth of $3 \mathrm{~cm}$ while the distance from the antenna to the ground surface is $12 \mathrm{~cm}$, so the distance between the antenna and the can is $15 \mathrm{~cm}$. The GPR is connected to the VNA through a bistatic Vivaldi antenna. VNA is configured to receive an electromagnetic wave from the receiver antenna at port 2. The data collected by the VNA is $S_{21}$. The GPR moves every $1 \mathrm{~cm}$ step and scans the target to obtain B-scan data.

\section{Result and Analysis}

When conducting the experiment, almost all pulse widths were tested. In this study, the duty cycles are chosen so that the images on the B-scan are varied from clear to invisible ones. 


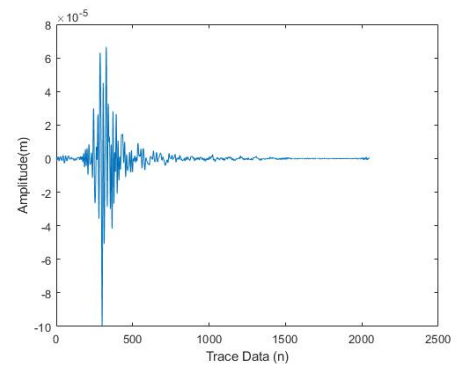

Fig. 5. A-scan using Ricker wavelet with $0.585 \%$ duty cycle

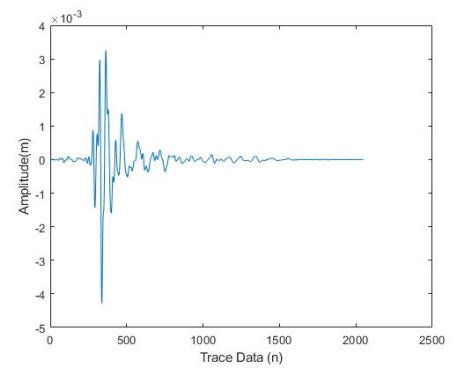

Fig. 6. A-scan using Ricker wavelet with $3.42 \%$ duty cycle

Fig. 5 reveals that the shape of the A-scan generated with the Ricker wavelet $0.585 \%$ duty cycle in a single period. In Fig. 6 shows the form of A-scan generated with Ricker Wavelet at a time of $3.42 \%$ duty cycle in one period. In Fig. 7 shows the form of A-scan generated with Ricker Wavelet at a time of $6.35 \%$ duty cycle in one period. In Fig. 8 shows that the A-scan generated with Ricker Wavelet at a time of $9.52 \%$ duty cycle in one period. The four images show the difference of each A-scan from the multiplication result between the echo signal and Ricker wavelet with different pulse width. The A-scan image cannot describe whether a pulse is good, because the shape of the target is not detected, so it is forwarded to the B-scan to see whether the target is detected or not.

In B-scan, the red circle is highlight to the buried target. In Fig. 9 reveals that the shape of the B-scan was generated with the Ricker wavelet $0.585 \%$ duty cycle in one period. It seems from the image that the target is still blurry and not yet visible. However, the image also shows portions of thin or medium clutter. In Fig. 10 shows that the form of B-scan was generated with Ricker Wavelet at a time of $3.42 \%$ duty cycle in one period. It can be seen from the picture that the target is clearly visible, with a very prominent arch. It also shows a thicker clutter in the figure. In Fig. 11 shows that the form of B-scan was generated with Ricker Wavelet at a time of $6.35 \%$ duty cycle in

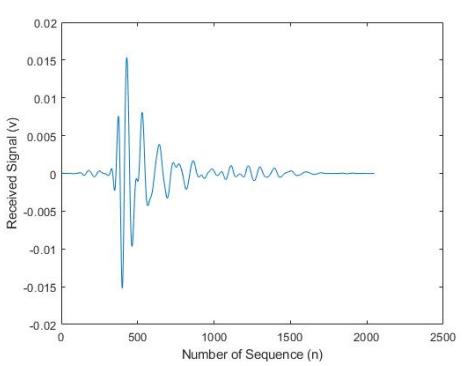

Fig. 7. A-scan using Ricker wavelet with $6.35 \%$ duty cycle

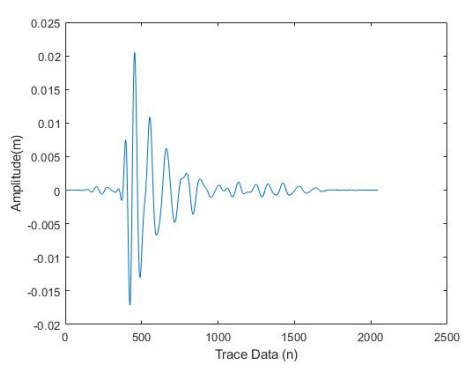

Fig. 8. A-scan using Ricker wavelet with $9.52 \%$ duty cycle

one period. The target picture seems fuzzy, so it's hard to see it. In Fig. 12 shows that the form of B-scan was generated with Ricker Wavelet at a time of $9.52 \%$ duty cycle in one period. It looks from the picture that the target is so blurred or even not visible that it's hard to see, and it's got a very thick clutter.

There are similarities in the effects of the images for each separate duty cycle in the A-scan and B-scan. If the duty cycle is higher, the A-scan will impact the larger pulse width. Targets are only visible on the B-scan, because there is an indentation of the target. The effect of the duty cycle can be seen in the thickness of the clutter, the higher the duty cycle, the thicker the clutter. When the duty cycle is high it will produce a wide

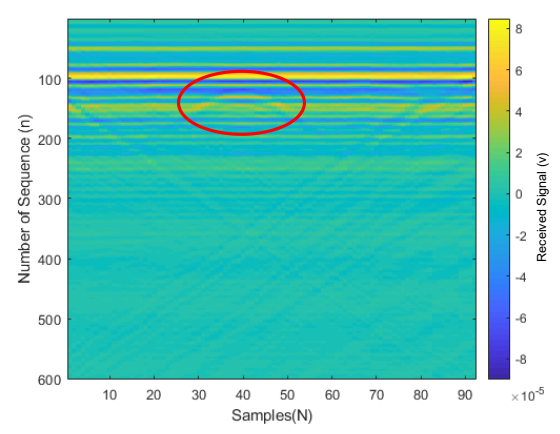

Fig. 9. B-scan using Ricker wavelet with $0.585 \%$ duty cycle 
Ramadhan et al / Journal of Measurement, Electronics and Communication Systems

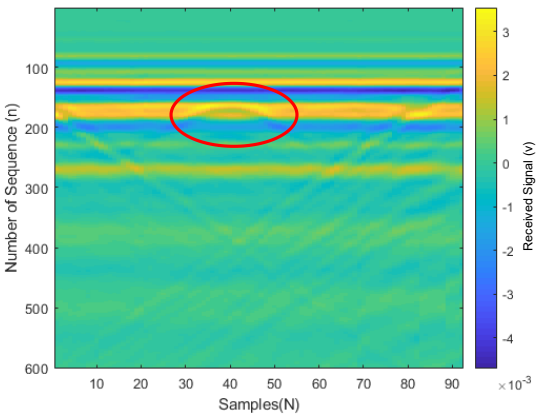

Fig. 10. B-scan using Ricker wavelet with $3.42 \%$ duty cycle

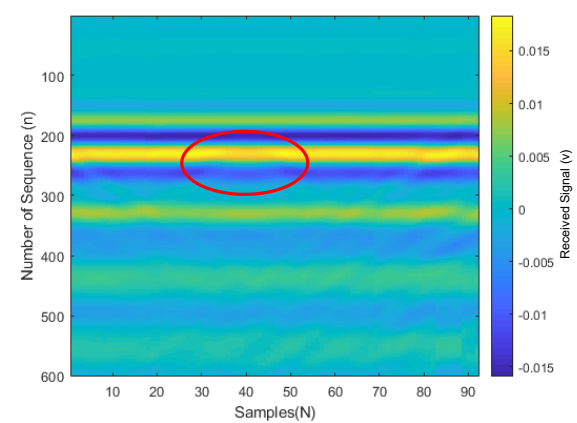

Fig. 11. B-scan using Ricker wavelet with $6.35 \%$ duty cycle

pulse width and produce a high resolution.

\section{Conclusion}

This paper concludes that the clarity of the buried object images are dependent on the duty cycles. This study found the best duty cycle is $3.42 \%$ of Ricker wavelet. For this experiment, it can also be seen that the larger the duty cycle the greater the received signal. If the duty cycle is too large, the target overwritten by the received signal so that it cannot be seen.

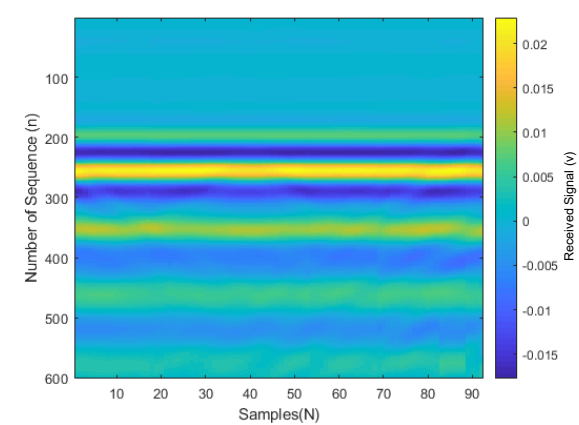

Fig. 12. B-scan using Ricker wavelet with $9.52 \%$ duty cycle

\section{References}

[1] David J. Daniels, "Ground Penetrating Radar," 2nd Edition, The Instution of Electrical Engineers, 2004.

[2] Lorenzo Crocco and Vincenzo Ferrara, "A Review On Ground Penetrating Radar Technology For The Detection Of Buried Or Trapped Victims," in International Conference on Collaboration Technologies and Systems (CTS), May 2014, pp. 535-540.

[3] Erfansyah Ali, A. A. Pramudita, Dharu Arseno, "Concrete Thickness Measurement Model for GPR," in IEEE Conference on Antenna Measurements Applications (CAMA), Bali-Indonesia, 2019.

[4] Qiang Li, Zhenghuan Xia, Jinjin Shao, Jie Chen, Xiaojun Liu, and Guangyou Fang, "Non-destructive Survey of Pavement Layer Thicknesses with Ground Penetrating Radar," in IEEE International Conference of IEEE Region 10 (TENCON 2013), October 2013, pp.1-4

[5] Raffaele Persico, "Introduction To Ground Penetrating Radar Inverse Scattering and Data Processing," Tariq Samad, Ed. IEEE Press, 2014.

[6] Varun Kumar and Subrata Maiti, "A Nobel Characterization of Shape of Pulse in GPR Signal Transmission," in International Conference on Communication and Signal Processing, April 2014, pp. 944-947.

[7] Afshin Gholamy and Vladik Kreinovich, "Why Ricker Wavelets Are Successful in Processing Seismic Data: Towards a Theoretical Explanation," in IEEE Symposium on Computational Intelligence for Engineering Solutions (CIES), December 2014, pp. 11-16.

[8] Yanghua Wang, "Geophysics," SEG Library, Vol 80, no. 2, ch. Frequencies of the Ricker wavelet, pp. A31-A37, March 2015.

[9] Hongliu Zeng, "How thin is a thin bed? An alternative perspective," in The Leading Edge Journal, Vol 28, no 10, pp. 1192-1197, October 2009.

[10] Erica Carrick Utsi, "Ground Penetrating Radar Theory and Practice," Ken McCombs, Ed. Joe Hayton, 2017.

[11] Harry M. Jol, "Ground Penetrating Radar Theory and Application," 1st ed. Elsevier B.V., 2009. 
[12] Yanghua Wang, "Seismic Inversion Theory and Applications," John Wiley, Ed. Wiley Blackwell, 2017.

[13] Nosherwan Shoaib, "Vector Network Analyzer(VNA) Measurements and Uncertainty Assessment," Springer International Publishing, 2017.

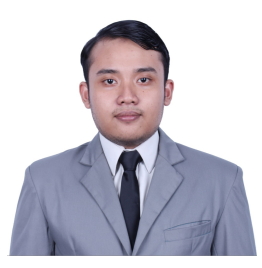

Aditya Rifky Ramadhan, received a bachelor's degree in telecommunication engineering from Telkom University in 2020. He is a researcher in Satellite Communication and Radar Laboratory in Electrical Power Subsystem Division. He is passionate Radar and Nanosatellite Satellite technology.

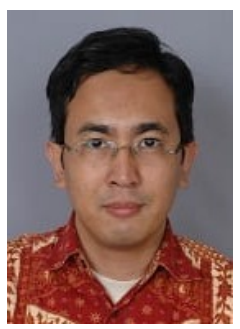

Erfansyah Ali, received his BS (2005) and MS (2012) both from Electrical Engineering Department major in Communications, Intitut Teknologi Bandung (ITB). Currently he is a lecturer at School of Electrical Engineering, Telkom University and a $\mathrm{PhD}$ student at National Taiwan University of Science and Technology (NTUST). His research interests are in Radar Systems, RF device and Signal Processing.

Aloysius Adya Pramudita (S'08 M'12) was born in Klaten, Indonesia, in 1977. He received the B.S. in electrical engineering from Gadjah Mada University, Indonesia in 2000, M.S. and Ph.D degree in electrical engineering from the Bandung Institute of Technology, Indonesia in 2005 and 2009 respectively. From 2002 to 2016, he was a Lecturer and Researcher with the Atma Jaya Catholic University, Indonesia. From 2013 to 2016, He was head of Electrical Engineering Department of Atma Jaya Univeristy and from 2016 to 2017 as head of Electrical Engineering Graduate Program of Atma Jaya Catholic University. Since 2017, he joined the Telecommunication Engineering Department of Telkom University, Bandung, Indonesia. He responsible as head of Satellite Communication and Radar Lab- oratory of Telkom University. He also serves as a reviewer for several technical journals and conferences in his interest area. His research interests include antenna theory and design for telecommunication and radar, electromagnetics and wave application, and radar system for contactless sensor 